\title{
Some multipliers on the space consisting of measures of analytic type, II
}

\author{
By Hiroshi Yamaguchi \\ (Received January 5, 1983)
}

\section{$\S$ 0. Introduction}

Let $G$ be a LCA group with the dual group $G . \quad m_{G}$ means the Haar measure of $G . \quad M(G)$ and $L^{1}(G)$ denote the measure algebra and the group algebra respectively. Let $M_{s}(G)$ be the closed subspace of $M(G)$ consisting of the singular measures on $G$. For a subset $E$ of $G, M_{E}(G)$ denotes the space of measures in $M(G)$ whose Fourier-Stieltjes transforms vanish off $E$. We denote by $E^{0}$ and $E^{-}$the interior and closure of $E$ respectively. "^", and " " denote the Fourier-Stieltjes transform and the inverse Fourier transform respectively. For a subset $B$ of $M(G), B^{\wedge}$ means a set $\{\hat{\mu}: \mu \in B\}$. Let $R$ be the reals and $H^{1}(R)$ the Hardy space on $R$. Then, by the $\mathrm{F}$. and M. Riesz theorem, $H^{1}(R)=\{\mu \in M(R): \hat{\mu}(x)=0$ for $x<0\}$. When there is a nontrivial continuous homomorphism $\phi: G \mapsto R$, we define $M^{a}(G)$ by $M^{a}(G)=\{\mu \in M(G): \hat{\mu}(\gamma)=0$ for $\gamma \in \hat{G}$ with $\phi(\gamma)<0\}$. If $\mu \in M^{a}(G)$, we say that $\mu$ is a measure of analytic type.

For compact abelian groups $G$, Doss proved that each multiplier on $M_{s}(G)$ is given by convolution with a discrete measure on $G$ ([3]). In [5], Graham and MaLean obtained an analogous result for LCA groups. On the other hand, the author in ([10], Theorem 2.3) proved that $\Phi \circ \psi$ becomes a multiplier on $M^{a}(G)$ for each multiplier $\Phi$ on $L_{-\delta}^{1}(R)(\delta>0)$, where $L_{-\delta}^{1}(R)$ $=\left\{f \in L^{1}(R): \hat{f}(x)=0\right.$ for $\left.x<-\delta\right\}$. However it is natural to consider whether $\Phi \circ \phi$ becomes a multiplier on $M^{a}(G)$ for each multiplier $\Phi$ on $H^{1}(R)$ or not. There are two purpose in this paper. One is to prove that $\Phi$ 。 $\phi$ becomes a multiplier on $M^{a}(G)$ for each multiplier $\Phi$ on $H^{1}(R)$. The other is to improve Theorem 2.4 in [10]. We state our results after the following definition.

Definition 0.1 Let $E$ be a aubset of $\hat{G}$. A function $\Phi$ on $\hat{G}$ rohich is continuous on $E^{0}$ is called a multiplier (or multiplier function) on $M_{E}(G)$ if $\Phi \hat{\mu} \in M_{E}(G)^{\wedge}$ for each $\mu \in M_{E}(G)$. By the function $\Phi$, there exists a unique bounded linear operator $S$ on $M_{E}(G)$ such that $S(\mu)^{\wedge}=\Phi \hat{\mu}$. Thus defined $S$ is called a multiplier operator (or merely multiplier) on $M_{E}(G)$ 
induced by the function $\Phi$. We denote a norm $\|\Phi\|$ by $\|\Phi\|=\|S\|$.

THEOREM I (cf. Theorem, 2.3 in [10]).

Let $G$ be a LCA group and $\psi$ a nontrivial continuous homomorphism from $G$ into $R$. Suppose $M^{a}(G) \cap M_{s}(G) \neq\{0\}$. Let $\Phi$ be a multiplier on $H^{1}(R)$. Then $\Phi \circ \phi$ is a multiplier on $M^{a}(G)$ with the following properties:

( I ) $S\left(M^{a}(G) \cap L^{1}(G)\right) \subset M^{a}(G) \cap L^{1}(G)$;

(II) $\quad S\left(M^{a}(G) \cap M_{s}(G)\right) \subset M^{a}(G) \cap M_{s}(G)$;

(III) $\quad|| \Phi \circ \psi \| \leqq|\Phi(0)|+2|| \Phi||$,

where $S$ is the bounded linear operator on $M^{a}(G)$ corresponding to $\Phi \circ \phi$.

TheOREM II (cf. Theorem 2.4 in [10]).

Let $G$ be a LCA group and $P$ a semigroup in $G$ such that $P \cup(-P)=\hat{G}$. We assume that $P$ is not dense in $G$ and $M_{P}(G) \cap M_{s}(G) \neq\{0\}$. Then there exists a multiplier $\Phi$ on $M_{P}(G)$ which satisfies the following:

( I ) $S\left(M_{P}(G) \cap L^{1}(G)\right) \subset M_{P}(G) \cap L^{1}(G)$;

(II) $\quad\{0\} \subsetneq S\left(M_{P}(G) \cap M_{s}(G)\right) \subset M_{P}(G) \cap M_{s}(G)$;

(III) $S$ is not given by convolution with a bounded regular measure on $G$,

where $S$ is the bounded linear operator on $M_{P}(G)$ corresponding to $\Phi$.

\section{$\S 1$. Some lemmas}

In this section we state lemmas which are needeful for the proofs of Theorems I and II. For a subgroup $\Lambda$ of $\hat{G}, \Lambda^{\perp}$ means the annihilator of $\Lambda$.

Lemma 1.1. Let $G$ be a metrizable LCA group and $P$ a semigroup in $\hat{G}$ such that $P \cup(-P)=G$. Put $\Lambda=P \cap(-P)$ and $H=\Lambda^{\perp}$. If $P$ is open, we have

$$
m_{H^{*}}\left\{M_{P}(G) \cap M_{s}(G)\right\} \subset M_{P}(G) \cap M_{s}(G) .
$$

Proof. We may assume that $P \subsetneq G$. First we consider the case that $G$ is $\sigma$-compact metrizable. Let $\mu$ be a measure in $M_{P}(G) \cap M_{s}(G)$. Put $\eta=\pi(|\eta|)$, where $\pi: G \mapsto G / H$ is the natural homomorphism. Then by the theory of disintegration (cf. [1], Théorème 1, p. 58) there exists a family $\left\{\xi_{\dot{x}}\right\}_{\dot{x} \in G / H}$ of positive measures in $M(G)$ with the following properties :

(1) $\quad \dot{x} \mapsto \xi_{\dot{x}}(f)$ is a Borel measurable function for each bounded Borel function $f$ on $G$;

(2) $\quad \operatorname{supp}\left(\xi_{\dot{x}}\right) \subset \pi^{-1}(\dot{x})$;

(3) $\quad\left\|\boldsymbol{\xi}_{\dot{x}}\right\| \leq 1$;

(4) $|\mu|(f)=\int_{G / H} \xi_{\dot{x}}(f) d \eta(\dot{x})$ for each bounded Borel $f$ on $G$. 
For $\dot{x} \in G / H, m_{\dot{x}}$ denotes the measure on $\pi^{-1}(\dot{x})$ which is given by translating $m_{H}$ to $\pi^{-1}(\dot{x})$. Let $\xi_{\dot{x}}=\xi_{\dot{x}}^{a}+\xi_{\dot{x}}^{s}$ be the Lebesgue's decomposition of $\xi_{\dot{x}}$ with respect to $m_{\dot{x}}$, where $\xi_{\dot{x}}^{a} \ll m_{\dot{x}}$ and $\xi_{\dot{x}}^{s} \perp m_{\dot{x}}$. Let $h$ be a unimodular Borel function on $G$ such that $\mu=h|\mu|$. We define measures $\lambda_{\dot{x}}, \lambda_{\dot{x}}^{a}$ and $\lambda_{\dot{x}}^{s}$ on $G$ as follows :

$$
\lambda_{\dot{x}}=h \xi_{\dot{x}}, \quad \lambda_{\dot{x}}^{a}=h \xi_{\dot{x}}^{a}, \quad \lambda_{\dot{x}}^{s}=h \xi_{\dot{x}}^{s} .
$$

Then, by (1)-(4) and ([11], Lemma 2.5), we have

(5) $\quad \dot{x} \mapsto \lambda_{\dot{x}}(f)$ is a Borel measurable function for each bounded Borel function $f$ on $G$;

(6) $\quad \operatorname{supp}\left(\lambda_{\dot{x}}\right) \subset \pi^{-1}(\dot{x})$;

(7) $\quad\left\|\lambda_{\dot{x}}\right\| \leq 1$;

(8) $\quad \mu(f)=\int_{G / H} \lambda_{\dot{x}}(f) d \eta(\dot{x})$ for each bounded Borel $f$ on $G$;

(9) $\quad \dot{x} \mapsto \lambda_{\dot{x}}^{a}(f)$ and $\dot{x} \mapsto \lambda_{\dot{x}}^{s}(f)$ are Borel measurable functions for each bounded Borel function $f$ on $G$.

Since $P$ is open, we note that $P$ is closed. Moreover, since $P^{c}+\Lambda=P^{c}$, we note that ([11], Lemma $2.4(5))$ is satisfied even if we replace $P$ by $P^{c}$. Hence, since $\mu \in M_{P}(G)$, we have

$$
\hat{\lambda}_{\dot{x}}(\gamma)=0 \quad \text { on } P^{c} \quad \eta \text {-a. a. } \dot{x} \in G / H .
$$

Then, by (6), (10) and ([8], 8.2.3. Theorem (b), p. 200), we have

$$
\lambda_{\dot{x}}^{s \wedge}(\gamma)=0 \quad \text { on }-P \quad \eta-\text { a. a. } \dot{x} \in G / H .
$$

By (5) and (9) we can define measures $\mu_{i} \in M(G)$ as follows :

$$
\begin{aligned}
& \mu_{1}(f)=\int_{G / H} \lambda_{\dot{x}}^{a}(f) d \eta_{a}(\dot{x}), \\
& \mu_{2}(f)=\int_{G / H} \lambda_{\dot{x}}^{s}(f) d \eta_{a}(\dot{x}), \\
& \mu_{3}(f)=\int_{G / H} \lambda_{\dot{x}}(f) d \eta_{s}(\dot{x})
\end{aligned}
$$

for $f \in C_{0}(G)$, where $\eta=\eta_{a}+\eta_{s}$ is the Lebesgue's decomposition of $\eta$ with respect to $m_{G / H}$. Then by ([11], Lemma 2.6, Claims 1-3) and the construction of $\mu_{i}$, we have $\mu_{1} \in L^{1}(G)$ and $\mu_{2}, \mu_{3} \in M_{s}(G)$. Since $\mu \in \mathrm{M}_{s}(G)$ and $\mu=\mu_{1}+\mu_{2}+\mu_{3}$, we get

$$
\mu=\mu_{2}+\mu_{3} \text {. }
$$

By (11) and the construction of $\mu_{2}$, we have $\hat{\mu}_{2}=0$ on $-P$, which yields 


$$
m_{H^{*}} * \mu_{2}=0 .
$$

Moreover we have

$$
m_{H^{*}} * \mu_{3} \in M_{s}(G) \text {. }
$$

Indeed, since $\pi\left(\left|\mu_{3}\right|\right) \leq \eta_{s}$, we have $\pi\left(m_{H} *\left|\mu_{3}\right|\right) \in M_{s}(G / H)$, hence $m_{H^{*}}\left|\mu_{3}\right| \in$ $M_{s}(G)$ (cf. [11], Lemma 2.3). Thus (14) is obtained. Hence, when $G$ is $\sigma$-compact metrizable, the lemma follows from (12)-(14).

Next we consider the case that $G$ is metrizable. Let $\mu$ be a measure in $M_{P}(G) \cap M_{s}(G)$. Then by ([11], Lemma 3.1) there exists a $\sigma$-compact open subgroup $G_{1}$ of $G$ such that

$$
\operatorname{supp}(\mu) \subset G_{1} \text { and } G_{1}^{\perp} \subset \Lambda .
$$

We note that $H \subset G_{1}$. Let $\tau$ be the natural homomorphism from $G$ onto $G / G_{1}^{\perp}$ and put $\tilde{P}=\tau(P)$. Then we have

$$
\tilde{\Lambda}^{\perp}=H \text {, }
$$

where $\tilde{\Lambda}=\tilde{P} \cap(-\tilde{P})$. By (15), we can regard $\mu$ as a measure in $M_{s}\left(G_{1}\right)$. Since $G_{1}^{\perp} \subset P \cap(-P)$, we have $\tau\left(P^{c}\right)=\tau(P)^{c}$. Hence $\mu$ belongs to $M_{\mathfrak{P}}\left(G_{1}\right) \cap$ $M_{s}\left(G_{1}\right)$. Since $G_{1}$ is $\sigma$-compact metrizable, it follows from (16) and the first half that $m_{H} * \mu \in M_{\mathfrak{P}}\left(G_{1}\right) \cap M_{s}\left(G_{1}\right)$. In particular, $m_{H} * \mu$ belongs to $M_{P}(G) \cap$ $M_{s}(G)$ and the proof is complete.

Lemma 1.2. Let $G$ be a LCA group and $P$ an open semigroup in $G$ such that $P \cup(-P)=G$. Then the previous lemma is also satisfied.

Proof. Let $\Lambda$ and $H$ be as in the previous lemma. Let $\mu$ be a measure in $M_{P}(G) \cap M_{s}(G)$. Suppose $m_{H} * \mu$ does not belong to $M_{P}(G) \cap M_{s}(G)$. Then $m_{H} * \mu=f+\nu$, where $\nu \in M_{s}(G)$ and $f$ is a nonzero function in $L^{1}(G)$. Since $\hat{f} \in C_{0}(G)$, there exists a $\sigma$-compact open subgroup $F$ of $G$ such that $\hat{f}(\gamma)=0$ for $\gamma \notin F$. Since $\mu, \nu \in M_{s}(G)$, there exist $\sigma$-compact subsets $E_{\mu}$ and $E_{\nu}$ of $G$ such that $|\mu|\left(E_{\mu}^{c}\right)=0,|\nu|\left(E_{\nu}^{c}\right)=0$ and $m_{G}\left(E_{\mu} \cup E_{\nu}\right)=0$. Hence by ([7], Lemma 4) there exists a $\sigma$-compact open subgroup $\Gamma$ of $G$ such that (a) $\Gamma \supset F$ and (b) $m_{G}\left(\Gamma^{\perp}+E_{\mu} \cup E_{\nu}\right)=0$. Let $\pi$ be the natural homomorphism from $G$ onto $G / \Gamma^{\perp}$. Then, by (b), we have

$$
\pi(\mu), \pi(\nu) \in M_{s}\left(G / \Gamma^{\perp}\right) .
$$

Put $P_{1}=P \cap \Gamma$. Then $P_{1}$ is an open semigroup in $\Gamma$ such that $P_{1} \cup\left(-P_{1}\right)=\Gamma$. Put $\Lambda_{1}=P_{1} \cap\left(-P_{1}\right)$, and let $\tilde{H}$ be the annihilator of $\Lambda_{1}$ in $G / \Gamma^{\perp}$. Then we have $\pi\left(m_{H}\right)=m_{\tilde{H}}$. Since $G / \Gamma^{\perp}$ is metrizable, it follows from (1) and Lemma 1. 1 that

$$
\pi\left(m_{H}\right) * \pi(\mu)=m_{\tilde{B}} * \pi(\mu) \in M_{P_{1}}\left(G / \Gamma^{\perp}\right) \cap M_{s}\left(G / \Gamma^{\perp}\right) .
$$


On the other hand, we have

$$
\pi\left(m_{H}\right) * \pi(\mu)=\pi\left(m_{H} * \mu\right)=\pi(\mu)+\pi(f) .
$$

By the construction of $\Gamma, \pi(f)$ is a nonzero function in $L^{1}\left(G / \Gamma^{\perp}\right)$. Hence by (1) and (2) we have a contradiction. Thus $m_{H} * \mu$ belongs to $M_{P}(G) \cap M_{s}(G)$ and the proof is complete.

PRoposition 1.3. Let $G$ be a LCA group and $P$ a semigroup in $G$ such that $P \cup(-P)=G$. Put $\Lambda=P^{-} \cap(-P)^{-}$and $H=\Lambda^{\perp}$. If $\Lambda$ is open, we have $m_{H} *\left\{M_{P}(G) \cap M_{s}(G)\right\} \subset M_{P}(G) \cap M_{s}(G)$.

Proof. If $\Lambda$ is open, $P^{-}$is an open semigroup in $G$ such that $P^{-} U$ $(-P)^{-}=\hat{G}$. Hence by Lemma 1.2 and the fact that $M_{P}(G) \subset M_{P^{-}}(G)$, the proposition is obtained.

\section{$\S$ 2. Proof of Theorem I.}

In this section we prove Theorem I.

Definition 2.1. Let $G$ be a LCA group and $\phi: G \mapsto R$ a nontrivial continuous homomorphism. Let $\delta$ be a positive real number. We define $M_{\delta}(G)$ as follows:

$$
M_{\delta}(G)=\{\mu \in M(G): \hat{\mu}(\gamma)=0 \text { for } r \in G \text { with } \phi(\gamma)<\delta\} .
$$

Let $\Phi$ be a multiplier on $H^{1}(R)$. Then $\Phi_{\delta}(x)=\Phi(x+\delta)$ becomes a multiplier on $L_{-\delta}^{1}(R)$. Hence the following lemma is obtained from ([10, Theorem 2.3).

Lemma 2.2. Let $G$ be a LCA group and $\psi: \hat{G} \mapsto R$ a nontrivial continuous homomorphism. Let $\delta$ be a positive real number and $\gamma_{\delta}$ an element in $G$ such that $\psi\left(\gamma_{0}\right)=\delta$. Let $\Phi$ be a multiplier on $H^{1}(R)$. Then $\Phi_{\circ} \phi$ is a multiplier on $M_{\delta}(G)$ satisfying the following:

( I) $S\left(M_{\delta}(G) \cap L^{1}(G)\right) \subset M_{\delta}(G) \subset L^{1}(G)$;

(II) $S\left(M_{\delta}(G) \cap M_{s}(G)\right) \subset M_{\delta}(G) \cap M_{s}(G)$;

(III) $\|\Phi \circ \phi\| \leqq\|\Phi\|$,

where $S$ is the bounded linear operator on $M_{\dot{\delta}}(G)$ corresponding to $\Phi \circ \psi$.

Lemma 2.3. Let $G$ be a LCA group and $\phi: G \mapsto R$ a nontrivial continuous homomorphism. Put $Q=\{\gamma \in G: \phi(\gamma)>0\}$. Let $\Phi$ be a multiplier on $H^{1}(R)$. Then $\Phi \circ \phi$ is a multiplier on $M_{Q}(G)$ with the following properties :

( I ) $S\left(M_{Q}(G) \cap L^{1}(G)\right) \subset M_{Q}(G) \cap L^{1}(G)$;

(II) $S\left(M_{Q}(G) \cap M_{s}(G)\right) \subset M_{Q}(G) \subset M_{s}(G)$;

(III) $\|\Phi \circ \phi\| \leq\|\Phi\|$, 
where $S$ is the bounded linear operator on $M_{Q}(G)$ corresponding to $\Phi \circ \phi$.

Proof. By Lemma 2.2, we may assume that $\phi(\hat{G)}$ is dense in $R$. Then we have

$$
\text { (*) } \quad \Phi \circ \phi \hat{\mu} \in M_{Q}(G)^{\wedge} \text { and }\|\Phi \circ \phi \hat{\mu}\| \leq\|\Phi\|\|\mu\| \text { for } \mu \in M_{Q}(G) \text {. }
$$

We can easily verify that $\Phi \circ \phi \hat{\mu}$ is continuous. Put $\Lambda=\operatorname{ker}(\phi)$, and let $p(x)=$ $\sum_{i=1}^{n} c_{i}\left(-x, \gamma_{i}\right)$ be a trigonometric polynomial on $G$. We consider (*) by dividing two cases that $\Lambda$ is open or not.

Case 1. $\Lambda$ is open.

Let $\varepsilon>0$ be any positive real number. Then by ([8], Theorem 2.6.8) there exists $\nu \in L^{1}(G)$ with the following properties :

$$
\hat{\nu}\left(\gamma_{i}\right)=1 \quad(1 \leq i \leq n) ;
$$

(2) $\hat{\nu}$ has a compact support ;

$$
\|\nu\| \leq 1+\varepsilon .
$$

Then, since $\Lambda$ is open, it follows from (2) that $\mu * \nu \in M_{\delta}(G)$ for some $\delta>0$. Hence by Lemma 2.2 and (3) we have $\Phi \circ \phi \hat{\mu} \hat{\nu} \in M_{Q}(G)^{\wedge}$ and $\|\Phi \circ \phi \hat{\mu} \hat{\nu}\| \leq$ $(1+\varepsilon)\|\Phi\|\|\mu\|$. Thus it follows from (1) that

$$
\begin{aligned}
\left|\sum_{i=1}^{n} c_{i} \Phi \circ \phi\left(\gamma_{i}\right) \hat{\mu}\left(\gamma_{i}\right)\right| & =\left|\sum_{i=1}^{n} c_{i} \Phi \circ \phi\left(\gamma_{i}\right) \hat{\mu}\left(\gamma_{i}\right) \hat{\nu}\left(\gamma_{i}\right)\right| \\
& \leq(1+\varepsilon)\|p\|_{\infty}\|\Phi\|\|\mu\| .
\end{aligned}
$$

Hence if $\Lambda$ is open, $\left(^{*}\right)$ follows from ([8], 1.9.1 Theorem) since $\varepsilon$ is any positive real number.

Case 2. $\Lambda$ is not open.

In this case, we note $G / \Lambda \cong R$. For any $\varepsilon>0$, we choose $\nu \in L^{1}(G)$ which satifies (1)-(3). Then $\mu * \nu \in L^{1}(G)$ and $\mu \hat{*}=0$ on $\Lambda$. Hence by ([8], Theorem 2.7.5) there exists $\xi \in M\left(\Lambda^{\perp}\right)$ with the following properties:

$$
\begin{aligned}
& \hat{\xi}=1 \text { on an open set containing } \Lambda ; \\
& \|\mu * \nu * \xi\|<\varepsilon .
\end{aligned}
$$

Then by (4) we have $\mu * \nu-\mu * \nu * \xi \in M_{\delta}(G)$ for some $\delta>0$. Hence by (5) and Lemma 2. 2 we have

$$
\begin{gathered}
\left|\sum_{i=1}^{n} c_{i} \Phi \circ \phi\left(\gamma_{i}\right) \hat{\mu}\left(\gamma_{i}\right)\right|=\left|\sum_{i=1}^{n} c_{i} \Phi \circ \phi\left(\gamma_{i}\right)(\mu * \nu)^{\wedge}\left(\gamma_{i}\right)\right| \\
\leq\left|\sum_{i=1}^{n} c_{i} \Phi \circ \psi\left(\gamma_{i}\right)(\mu * \nu-\mu * \nu * \xi)^{\wedge}\left(\gamma_{i}\right)\right| \\
+\left|\sum_{i=1}^{n} c_{i} \Phi \circ \phi\left(\gamma_{i}\right)(\mu * \nu * \xi)^{\wedge}\left(\gamma_{i}\right)\right|
\end{gathered}
$$




$$
\begin{aligned}
& \leq\|\Phi\|\|\mu * \nu-\mu * \nu * \xi\|\|\| p\left\|_{\infty}+\left(\sum_{i=1}^{n}\left|c_{i}\right|\right)\right\| \Phi\|\| \mu * \nu * \xi \| \\
& \leq\|\Phi\|\|p\|_{\infty}\{(1+\varepsilon)\|\mu\|+\varepsilon\}+\varepsilon\left(\sum_{i=1}^{n}\left|c_{i}\right|\right)\|\Phi\| .
\end{aligned}
$$

Letting $\varepsilon \downarrow 0$, then $\left(^{*}\right)$ follows from ([8], 1.9.1 Theorem). Thus our claim is satisfied. $\mathrm{By}(*), \Phi \circ \psi$ is a multiplier on $M_{Q}(G)$ and (III) is obtained. (I) follows from ([10], Lemma (E), p. 175). Finally we prove (II). For each $n \in N$, let $u_{n}$ be a function in $L^{1}(R)$ such that $\hat{u}_{n}(x)=1$ for $|x| \leq \frac{1}{n}$ and $\hat{u}_{n}(x)=0$ for $|x| \geq \frac{2}{n}$. Let $\phi: R \mapsto G$ be the dual homomorphism of $\phi$, and put $\xi_{n}=\phi\left(u_{n}\right)$. We define bounded linear operators $U_{n}$ on $M_{Q}(G)$ by

$$
U_{n}(\eta)=\eta-\eta * \xi_{n}
$$

Let $\mu \in M_{Q}(G) \cap M_{s}(G)$. We note

$$
U_{n}\left((\Phi \circ \phi \hat{\mu})^{\vee}\right)=\left(\Phi \circ \phi U_{n}(\mu)^{\wedge}\right)^{\vee} \quad(n=1,2,3, \cdots) .
$$

Suppose $(\Phi \circ \psi \hat{\mu})^{\vee}=\omega+f$, where $\omega \in M_{s}(G)$ and $f$ is a nonzero function in $L^{1}(G)$. Then by ([10], Theorem 4.1) and Lemma 1.2 we have $\omega, f \in M_{Q}(G)$. Hence there exists a positive integer $m$ such that $U_{m}(f) \neq 0$. By ([10], Theorem 2.3), we note $U_{m}$ maps $M_{Q}(G) \cap M_{s}(G)$ into itself. Hence we have

$$
\begin{aligned}
U_{m}\left((\Phi \circ \phi \hat{\mu})^{\curlyvee}\right) & =U_{m}(\omega)+U_{m}(f) \\
& \notin M_{s}(G) .
\end{aligned}
$$

On the other hand, since $U_{m}(\mu) \in M_{\frac{1}{m}}(G) \cap M_{s}(G)$, it follows from Lemma 2.2 that $\Phi \circ \phi U_{m}(\mu)^{\wedge} \in M_{s}(G)^{\wedge}$. This contradicts (7) and (8), and the proof is complete.

Now we prove Theorem I. First we consider the case that $\operatorname{ker}(\phi)$ is not open. In this case, we note $M^{a}(G)=M_{Q}(G)$, where $Q=\psi^{-1}((0, \infty))$. Hence the theorem follows from Lemma 2.3. Next we consider the case that $\operatorname{ker}(\psi)$ is open. Let $H=\operatorname{ker}(\psi)^{\perp}$, and we define a bounded linear operator $U: M^{a}(G) \mapsto M_{Q}(G)$ by $U(\mu)=\mu-\mu * m_{H}$. Then, for $\mu \in M^{a}(G)$, we have

$$
\Phi \circ \phi(\gamma) \hat{\mu}(\gamma)=\Phi(0)\left(\mu * m_{H}\right)^{\wedge}(\gamma)+\Phi \circ \phi(\gamma) U(\mu)^{\wedge}(\gamma) .
$$

Hence, by Lemmas 1.2 and 2.3 , we can verify that $\Phi \circ \psi$ is a multiplier on $M^{a}(G)$ which satisfies (I) and (II). Moreover, by (1), Lemma 2.3 and the construction of $U$, we have $\|\Phi \circ \psi\| \leq|\Phi(0)|+2\|\Phi\|$, hence (III) is obtained. This completes the proof.

\section{§ 3. Proof of Theorem II}

In this section we prove Theorem II. 
Lemma 3.1. Let $\Gamma$ be a LCA group with dual $G$. Suppose $P$ is a proper closed semigroup in $\Gamma$ such that (i) $P \cup(-P)=\Gamma$, (ii) $P \cap(-P)=\{0\}$ and (iii) $P$ induces a nonarchimedean order on $\Gamma$. Then there exists an open subgroup $\Lambda$ of $\Gamma$ with the following properties:

( I ) $\Lambda=(\Lambda+P) \cap(\Lambda-P)$;

(II) $M_{P \cap A}\left(G / \Lambda^{\perp}\right) \cap M_{s}\left(G / \Lambda^{\perp}\right) \neq\{0\}$;

(III) there exists a nontrivial continuous homomorphism $\phi: \Lambda \mapsto R$ such that $\psi^{-1}([0, \infty)) \supset P \cap \Lambda$.

Proof. We consider the lemma by dividing two cases that $\Gamma$ is discrete or not.

Case 1. $\Gamma$ is discrete.

In this case, there exist $\gamma_{1}, \gamma_{2} \in P \backslash\{0\}$ such that $n \gamma_{1}<\gamma_{2}$ for all $n \in Z$ (the integers). We define $S_{1}$ and $S_{2}$ as follows:

$$
\begin{aligned}
& S_{1}=\left\{\gamma \in P: n \gamma<\gamma_{2} \text { for all } n \in Z\right\} ; \\
& S_{2}=\left\{\begin{array}{c}
\mid \gamma \in P: n \gamma>\gamma_{2} \text { for some } n \in N \text { and } \\
\gamma<m \gamma_{2} \text { for some } m \in N
\end{array}\right\} \cup\{0\} .
\end{aligned}
$$

Put $\Lambda_{1}=S_{1} \cup\left(-S_{1}\right)$ and $\Lambda=\left(\Lambda_{1}+S_{2}\right) \cup\left(\Lambda_{1}-S_{2}\right)$. Then it is easy to see that $\Lambda_{1}$ and $\Lambda$ are subgroups of $\Gamma$. We show that $\Lambda$ is the desired one. First we prove $(\mathrm{I})$. Let $r$ be an element in $(\Lambda+P) \cap(\Lambda-P)$. We may assume that $\gamma \in P$. Suppose $\gamma \notin \Lambda$. Then we have

$$
\gamma>n \gamma_{2} \text { for all } n \in Z \text {. }
$$

Since $\gamma \in \Lambda-P$, there exist $\xi \in \Lambda$ and $p \in P$ such that $\gamma=\xi-p$. Then $\xi \geq \gamma$. Since $\xi \in \Lambda$, there exists a positive integer $m$ such that $m \gamma_{2}>\xi \geq \gamma$. This contradicts (1). Thus we have $(\Lambda+P) \cap(\Lambda-P) \subset \Lambda$. The converse inclusion is easily obtained. (II) is easily obtained from the construction of $\Lambda$. Next we prove (III). Let $\pi: \Lambda \mapsto \Lambda / \Lambda_{1}$ be the natural homomorphism. Then

$$
\pi(P \cap \Lambda) \text { induced an archimedean order on } \Lambda / \Lambda_{1} \text {. }
$$

In fact, it is easy to see that $\pi(\mathrm{P} \cap \Lambda)$ is a semigroup in $\Lambda / \Lambda_{1}$ such that $\pi(P \cap \Lambda)$ induces a total order on $\Lambda / \Lambda_{1}$. Let $\pi\left(\xi_{1}\right)$ and $\pi\left(\xi_{2}\right)$ be nonzero elements in $\pi(P \cap \Lambda)$. We may assume $\xi_{1}, \xi_{2} \in S_{2} \backslash\{0\}$. Then there exist positive integers $n_{1}$ and $n_{2}$ such that $n_{1} \gamma_{2}>\xi_{1}$ and $n_{2} \xi_{2}>\gamma_{2}$. Hence we have

$$
\begin{aligned}
2 n_{1} n_{2} \xi_{2} & =n_{1} n_{2} \xi_{2}+n_{1} n_{2} \xi_{2} \\
& >n_{1} \gamma_{2}+n_{1} n_{2} \xi_{2} \\
& >\xi_{1}+\gamma_{2} .
\end{aligned}
$$


Thus we have $2 n_{1} n_{2} \pi\left(\xi_{2}\right)>\pi\left(\xi_{1}\right)$, and (2) is obtained. By (2), there exists an order preserving isomorphism $\alpha$ from $\Lambda / \Lambda_{1}$ into $R$. We define $\phi: \Lambda \mapsto R$ by $\phi=\alpha \circ \pi$. Then we can verify that $\phi$ satisfies (III).

Case 2. $\Gamma$ is not discrete.

By [[8], 8.1.5 Theorem), we have

$$
\Gamma \cong R \oplus D \text {, }
$$

where $D$ is a nontrivial discrete ordered group. We note

$$
P \cong\{(x, d) \in R \oplus D: d>0 \text {, or } d=0 \text { and } x \geq 0\} .
$$

Let $d>0$ be an element in $D$, and put $\gamma_{2}=(0, d)$. For this $\gamma_{2} \in P \backslash\{0\}$, we construct subgroups $\Lambda_{1}$ and $\Lambda$ of $\Gamma$ as in Case 1 . Then $\Lambda$ is an open subgroup of $\Gamma$ which satisfies (I) and (II). Moreover, by the similar argument in Case 1, we can verify that (III) is satisfied. This completes the proof.

Lemma 3.2. Let $G$ be a LCA group and $P$ a semigroup in $G$ such that $P \cup(-P)=\hat{G}$ and $M_{P}(G) \cap M_{s}(G) \neq\{0\}$. If there exists a nontrivial continuous homomorphism $\psi: \hat{G} \mapsto R$ such that $\psi^{-1}([0, \infty)) \supset P$. Then there exists a multiplier $\Phi$ on $M_{P}(G)$ with the following properties:

$$
\{0\} \subsetneq S\left(M_{P}(G) \cap M_{s}(G)\right) \subset M_{P}(G) \cap M_{s}(G) ;
$$

$S$ is not given by convolution with a bounded regular measure on $G$,

where $S$ is the bounded linear operator on $M_{P}(G)$ corresponding to $\Phi$.

Proof. This follows from ([8], Theorem 2.4) and its proof.

Now we prove Theorem II. Put $F=P^{-} \cap(-F)^{-}$. Let $\pi: \hat{G} \mapsto \hat{G} / F$ be the natural homomorphism. Then $\pi\left(P^{-}\right)$is a proper closed semigroup in $\hat{G} / F$ such that (i) $\pi\left(P^{-}\right) \cup\left(-\pi\left(P^{-}\right)\right)=\hat{G} / F$ and (ii) $\pi\left(P^{-}\right) \cap\left(-\pi\left(P^{-}\right)\right)=\{0\}$. We consider the theorem by dividing two cases that $\pi\left(P^{-}\right)$induces an archimedean order on $\hat{G} / F$ or not.

Case 1. $\pi\left(P^{-}\right)$induces an archimedean order on $\hat{G} / F$.

In this case, by ([8], Theorems 8.1 .2 and 8.1 .6$, p. 194 and 196), there exists exists a nontrivial continuous homomorphism $\phi_{1}: G / F \mapsto R$ such that $\psi^{-1}([0, \infty))=\pi\left(P^{-}\right)$. Put $\phi=\psi_{1} \circ \pi$. Then $\phi: \hat{G} \mapsto R$ is a nontrivial continuous hcmomorphism such that $\psi^{-1}([0, \infty)) \supset P$. Hence by Lemma 3.2 there exists a multiplier $\Phi$ on $M_{P}(G)$ which satisfies (II) and (III) of the theorem. (I) follows from ([10], Lemma (E), p. 175). 
Case 2. $\pi\left(P^{-}\right)$induces a nonarchimedean order on $G / F$.

By Lemma 3.1 there exist an open subgroup $\tilde{\Lambda}$ of $G / F$ and a nontrivial continuous homomorphism $\tilde{\phi}: \tilde{\Lambda} \mapsto R$ with the following properties :

$$
\begin{aligned}
& \tilde{\Lambda}=\left(\tilde{\Lambda}+\pi\left(P^{-}\right)\right) \cap\left(\tilde{\Lambda}-\pi\left(P^{-}\right)\right) ; \\
& M_{\pi\left(P^{-}\right) \cap \tilde{\Lambda}}(\hat{\tilde{\Lambda}}) \cap M_{s}(\hat{\tilde{\Lambda}}) \neq\{0\} ; \\
& \tilde{\phi}^{-1}([0, \infty)) \supset \pi\left(P^{-}\right) \cap \tilde{\Lambda} .
\end{aligned}
$$

Put $\Lambda=\pi^{-1}(\tilde{\Lambda})$ and $\phi=\tilde{\phi} \circ \pi$. Then $\phi: \Lambda \mapsto R$ is a nontrivial continuous homomorphism, and it follows from (1)-(3) that

$$
\begin{aligned}
& \Lambda=\left(\Lambda+P^{-}\right) \cap\left(\Lambda-P^{-}\right) ; \\
& M_{P^{-} \cap \Lambda}(G / H) \cap M_{8}(G / H) \neq\{0\} ; \\
& \psi^{-1}([0, \infty)) \supset P^{-} \cap \Lambda,
\end{aligned}
$$

where $H$ is the annihilator of $\Lambda$. We note $P^{-} \cap \Lambda \subsetneq \Lambda$. Put $P_{\Lambda}=P \cap \Lambda$. Then by (5) we have

$$
M_{P_{A}}(G / H) \cap M_{s}(G / H) \neq\{0\} .
$$

We define a bounded linear operator $S_{1}: M_{P}(G) \mapsto M_{P_{A}}(G)$ by $S_{1}(\mu)=\mu * m_{H}$. Then, since $\Lambda+P$ is an open semigroup in $G$ with $(\Lambda+P) \cup(\Lambda-P)=G$, it follows from (4) and Lemma 1. 2 that

$$
S_{1}\left(M_{P}(G) \cap M_{s}(G)\right)=M_{P_{A}}(G) \cap M_{s}(G) .
$$

By (6), (7) and Lemma 3, 2, there exists a multiplier $\Phi_{0}$ on $M_{P_{A}}(G / H)$ with the following properties:

$$
\{0\} \subsetneq S_{\Phi_{0}}\left(M_{P_{\Lambda}}(G / H) \cap M_{s}(G)\right) \subset M_{P_{\Lambda}}(G / H) \cap M_{s}(G / H) ;
$$

(10) $S_{\Phi_{0}}$ is not given convolution with a bounded regular measure on $G / H$,

where $S_{\phi_{0}}$ is the bounded linear operator on $M_{P_{A}}(G / H)$ corresponding to $\Phi_{0}$. We define bounded linear operators $S_{2}: M_{\Lambda}(G) \mapsto M(G / H)$ and $S_{3}$ : $M(G / H) \mapsto M(G)$ as follows :

$$
\begin{aligned}
& S_{2}(\mu)^{\wedge}(\gamma)=\hat{\mu}(\gamma) \quad \text { for } \quad \gamma \in \Lambda \text {; } \\
& S_{3}(\nu) \wedge(\gamma)=\left\{\begin{array}{cl}
\hat{\nu}(\gamma) & \text { for } r \in \Lambda \\
0 & \text { for } r \notin \Lambda .
\end{array}\right.
\end{aligned}
$$


Now we define a bounded linear operator $S$ on $M_{P}(G)$ by $S=S_{3} \circ S_{\Phi_{0}} \circ S_{2} \circ S_{1}$ (see Figure I).

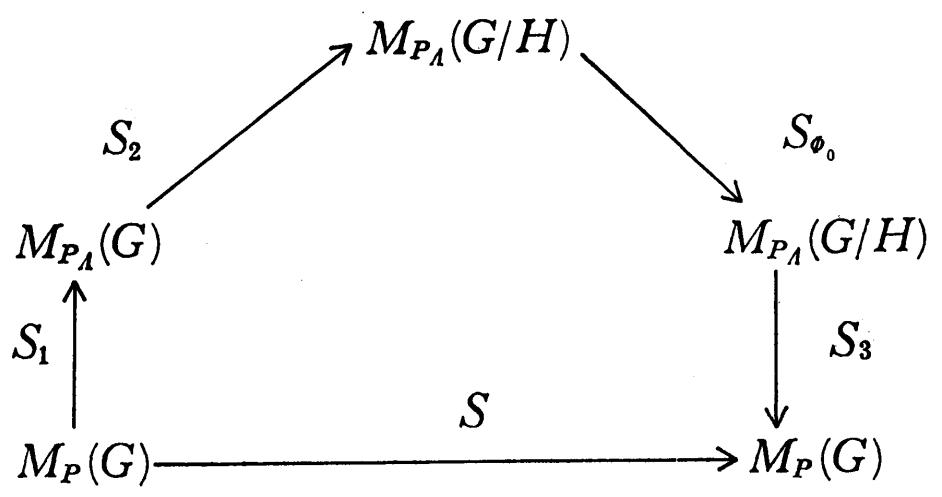

Fig. 1.

Then we have $S(\mu)^{\wedge}(\gamma)=\Phi(\gamma) \hat{\mu}(\gamma)$ for $\mu \in M_{P}(G)$, where $\Phi$ is a function on $G$ such that $\Phi(\gamma)=\Phi_{0}(\gamma)$ for $\gamma \in \Lambda$ and $\Phi(\gamma)=0$ for $\gamma \notin \Lambda$. Hence $\Phi$ is a multiplier on $M_{P}(G)$ corresponding to $S$. (I) of the theorem follows from $([10]$, Lemma $(E))$. By (7)-(9) and ([10], Lemmas (B) and (C), p. 174), we can verify that (II) is satisfied. Moreover (III) follows from (10). This completes the proof.

Finally the author wishes to express his thanks to Professor J. Inoue for his valuable advice.

\section{References}

[1] N. Bourbaki: Intégration, Éléments de Mathematique, Livre VI, Ch 6, Paris, Herman, 1959.

[2] K. DeLEeUW and I. GLICKSBERG: Quasi-invariance and analyticity of measures on compact groups, Acta. Math, 109 (1963), 179-205.

[ 3 ] R. Doss: Convolution of singular measures, Studia Mathematics, XLV (1973), 111-117.

[4] I. GLICKSBERG: Fourier-Stieltjes transforms with small supports, Illinois. J. Math, Vol 9 (1965), 418-427.

[5] C. C. Graham and A. MacLean: A multiplier theory for continuous measures, Studia Mathematics, LXVI (1980), 213-225.

[6] E. HewitT and K. A. Ross: Abstract Harmonic Analysis, Vol I and II, Springer-Verlag, New York-Heidelberg-Berlin, 1963 and 1970.

[7] L. PIGNO and S. SAEKI: Fourier-Stieltjes transforms which vanish at infinity, Math. Z, 141 (1975), 83-91.

[8] W. RudiN : Fourier Analysis On Groups, Interscience, New York, 1962.

[9] H. Yamaguchi: Some multiplierson $H_{P}^{1}(G)$, J. Austral. Math. Soc, (Series A) 29 (1980), 52-60. 
[10] H. YAMAGUCHI: Some multipliers on the space consisting of measures of analytic type, Hokkaido. Math. J, Vol XI (1982), 173-200.

[11] H. YAmAGUCHI: A property of some Fourier-Stieltjes Transforms. (to appear in Pacific. J. Math.)

Department of Mathematics, Josai University 